\title{
KALB-RAMOND COUPLED VORTEX FIBRATION MODEL FOR RELATIVISTIC SUPERFLUID DYNAMICS
}

\author{
Brandon Carter and David Langlois \\ Département d'Astrophysique Relativiste et de Cosmologie, \\ Centre National de la Recherche Scientifique, \\ Observatoire de Paris, 92195 Meudon, France \\ and \\ The Racah Institute of Physics, The Hebrew University, \\ Givat Ram, Jerusalem 91904, Israel
}

1st June 1995

\begin{abstract}
.
The macroscopic dynamics of a rotating superfluid deviates from that of a simple perfect fluid due to the effect of vorticity quantisation, which gives rise to a substructure of cosmic string type line defects that results in a local anisotropy whereby the effective average pressure in the direction of the vortex lines is reduced below its value in lateral directions. Whereas previous descriptions of this effect have been restricted to a non-relativistic framework that is adequate for the treatment of liquid helium in a laboratory context, the present work provides a fully relativistic description of the kind required for application to rotating neutron star models. To start with, the general category of vortex fibration models needed for this purpose is set up on the basis of a Kalb-Ramond type variational principle. The appropriate specification of the particular model to be chosen within this category will ultimately be governed by the conclusions of microscopic investigations that have not yet been completed, but the results available so far suggest that a uniquely simple kind of model with an elegant dilatonic formulation should be tentatively adopted as a provisional choice so long as there is no indication that a more complicated alternative is needed.
\end{abstract}




\section{Introduction.}

Until now, calculations of the macroscopic effect of quantised vortices in the superfluid layers of neutron stars have relied on the use of non relativistic formalism whose most complete and detailed development is due to Lindblom and Mende ${ }^{[1]}$. Despite the fact that relativistic corrections are by no means small, their neglect has so far been physically justifiable in the analysis of the most important observational effect for which they are relevant, namely pulsar frequency glitches, due to the many major uncertainties that are involved in the vortex pinning model that has been proposed as an explanation ${ }^{[2]}$. Although there is not yet any urgent need for the higher accuracy that it can provide, the use of a fully relativistic formulation is already wanted in order to satisfy a more pressing requirement, namely that of compatibility with the fully relativistic fluid models which have long been in standard use for representing the the gross structure of neutron stars: the main drawback of the mongrel combinations of relativistic and non relativistic elements grafted together in the treatments that have been used so far is not so much their lack of physical accuracy, but rather their lack of mathematical coherence which makes them very unweildy to work with. The purpose of the present work is to overcome this disadvantage by developing a more conveniently coherent approach in which the macroscopic effect of vortex quantisation is allowed for in a fully relativistic framework. The use of this approach is exemplified here by the introduction of a simple prototype model that is the natural relativistic generalisation of the simplest special case within the category of non-relativistic models considered by Lindblom and Mendel ${ }^{[1]}$, allowing only for the bulk motion of the neutron matter and ignoring the independent motion of the residual protons, whose analysis is postponed for future work of a more detailed nature.

The concepts on which the present approach is based were originally developed not in neutron star theory but in a more exotic cosmological context, specifically that of axion field theory. It was Lund and Regge ${ }^{[3]}$ who first drew attention to the analogy between Kalb-Ramond coupled string motion in an axion field and vortex motion in an ordinary non relativistic superfluid. The immediate inspiration for the coherent relativistic theory presented here was provided by the more recent analysis of Davis and Shellard ${ }^{[4][5]}$, and its modification to allow for more general kinds of field by Ben Ya'acov ${ }^{[6][7]}$. The outcome effectively extends and completes the results of an earlier pionneering investigation using a different but ultimately related approach by Lebedev and Khalatnikov ${ }^{[8]}$. 


\section{The perfect fluid limit.}

The proposed theory is obtained as the natural generalisation of an appropriately reformulated version of ordinary "barytropic" perfect fluid theory given ${ }^{[9]}$ by a Lagrangian variation principle which will now be described.

By definition, perfect (relativistic) fluid medium is characterised by a stress momentum energy density tensor $T^{\mu \nu}$ that is expressible in terms of its timelike unit eigenvector $u^{\mu}$, by the familiar formula $T^{\mu \nu}=\rho u^{\mu} u^{\nu}+P\left(g^{\mu \nu}+u^{\mu} u^{\nu}\right)$, with $u^{\mu} u_{\mu}=-1$ where $g_{\mu \nu}$ denotes the background metric, and the scalars $\rho$ and $P$ are respectively the relativistic mass-energy density and the pressure. The particularly simple case of an ordinary "barytropic" perfect fluid model is specifiable by an "equation of state" that determines $\rho$ as a function of the number density $n$ of a conserved current, or equivalently, in conjugate form, that determines $P$ as a function of the relativistic chemical potential or "effective mass" per particle variable $\mu$. The mutually conjugate functions for the mass density and the pressure are not independent but are symmetrically related by a Legendre type transformation given by

$$
\rho\{n\}+P\{\mu\}=n \mu, \quad \mu=\frac{d \rho}{d n}, \quad n=\frac{d P}{d \mu} .
$$

These functions determine the corresponding sound speed, $c_{\mathrm{S}}$ say, by a formula of the familiar form

$$
c_{\mathrm{S}}^{2}=\frac{d P}{d \rho}=\frac{n}{\mu} \frac{d \mu}{d n} .
$$

In such a model, as also on the generalisation to be presented in the next section, a particularly important role is played by the vorticity 2 -form $w_{\mu \nu}$, which is defined as the exterior derivative

$$
w_{\mu \nu}=2 \nabla_{[\mu} \mu_{\nu]}
$$

of the relevant 4-momentum covector, $\mu_{\lambda}$, which is the dynamical conjugate of the conserved particle current vector $n^{\lambda}$. The momentum covector is related to its dual, the corresponding momentum trivector, by

$$
\mu_{\lambda}=\frac{1}{3 !} \varepsilon_{\lambda \mu \nu \rho} H^{\mu \nu \rho}
$$

where $\varepsilon_{\lambda \mu \nu \rho}$ is the alternating tensor of the 4-dimensional spacetime background, while similarly the conserved current vector is given in terms of its dual 3-form $N_{\mu \nu \rho}$ by

$$
n^{\lambda}=\frac{1}{3 !} \varepsilon^{\lambda \mu \nu \rho} N_{\mu \nu \rho}
$$

The condition that the current is conserved, i.e. $\nabla_{\mu} n^{\mu}=0$, is expressible (in terms of covariant derivation with respect to the (flat or curved) spacetime metric $g_{\mu \nu}$ ) as the closure of the corresponding 3 -form, i.e.

$$
\nabla_{[\mu} N_{\nu \rho \sigma]}=0
$$

(where the square brackets denote index antisymmetrisation). 
In the particular case of the barytropic perfect fluid model, though not in the generalisation to be given in the following section, the momentum covector and its dynamical conjugate the current vector will be expressible in terms of the quantities introduced in (2.1) simply by

$$
\mu_{\lambda}=\mu u_{\lambda}, \quad n^{\lambda}=n u^{\lambda},
$$

which implies

$$
H^{\mu \nu \lambda}=\frac{\mu}{n} N^{\mu \nu \lambda}
$$

In this case the complete set of equations of motion is given by supplementing the kinematic relation (2.6) by a dynamical momentum transport equation which is obtainable from a convective variational principle ${ }^{[9]}$ in the form

$$
H_{\mu \nu \rho} \nabla_{\lambda} H^{\lambda \nu \rho}=0
$$

whose more familar dual form is

$$
n^{\mu} w_{\mu \nu}=0
$$

The most familiar category of equations of state to which this theory can be applied is the "polytropic" power law kind, $\rho \propto n^{\gamma}$, for a constant "polytropic index" $\gamma$. Particularly important examples are the pressure free ("dust") case, $\gamma=1$ (for which the flow lines are geodesics), and the ultrarelativistic (massless particle) gas case, $\gamma=4 / 3$. In the generic case the variable $\mu$ will not vary proportionally to $n$ so that the momentum 3 -vector $H^{\mu \nu \rho}$ will not be simply identifiable with the index raised version $N^{\mu \nu \rho}$ of the current 3-form $N_{\mu \nu \rho}$. However such an identification will be possible in the special case of the "stiff" (linear) Zel'dovich equation of state ${ }^{[10]}$, with $\gamma=2$, as given by

$$
\rho=\frac{\hbar^{3}}{12 m^{2}} N_{\mu \nu \rho} N^{\mu \nu \rho}, \quad H_{\mu \nu \rho}=\frac{\hbar^{3}}{m^{2}} N_{\mu \nu \rho},
$$

where $m$ is a fixed mass scale (exact identification being obtainable by choosing units such that $\left.m^{2}=\hbar^{3}\right)$.

For an arbitrary equation of state there is a corresponding (zero temperature) superfluid model $^{[11]}$ included as a particular application of the foregoing formalism when the (automatically conserved) vorticity is zero, since by (2.3) and (2.4) we shall then have

$$
w_{\mu \nu}=0 \Rightarrow \nabla_{\rho} H^{\rho \mu \nu}=0
$$

which is the necessary and locally sufficient integrability condition for the existence of a gauge scalar $\varphi$ that satisfies

$$
\mu_{\nu}=\nabla_{\nu}(\hbar \varphi) \Rightarrow H^{\mu \nu \rho}=\hbar \varepsilon^{\mu \nu \rho \sigma} \nabla_{\sigma} \varphi
$$

and that represents a condensate order parameter which may be presumed to be axionic in the sense of being periodic, so that it may have string-like topological defects. The normalisation used here is such that periodicity $2 \pi$ is consistent with the usual quantisation condition that the conserved circulation integral,

$$
\kappa=\oint \mu_{\nu} d x^{\nu}
$$


round a defect should be a multiple of the standard Bohr unit $2 \pi \hbar$. In the special case of a Zel'dovich fluid the corresponding superfluid model reduces exactly to the standard massless (Goldstone boson) axion field model ${ }^{[12][13][14]}$ with the coefficient $m$ representing the corresponding Higgs mass scale.

\section{The vortex dynamical generalisation.}

A Kalb-Ramond field is definable, modulo addition of the exterior derivative of an arbitrary 1-form $\alpha_{\mu}$, for any (perfect or other) conserved fluid as a gauge 2-form $B_{\mu \nu}$ from which the physical current 3 -form is obtainable by exterior differentiation, i.e.

$$
N_{\mu \nu \rho}=3 \nabla_{[\mu} B_{\nu \rho]}, \quad B_{\mu \nu} \cong B_{\mu \nu}+2 \nabla_{[\mu} \alpha_{\nu]},
$$

the closure property (2.6) being the Poincaré integrability condition that is necessary and locally sufficient for its existence. One can use it for setting up an appropriate action principle in which $B_{\mu \nu}$ is to be treated as a free field with the 2 -form $w_{\mu \nu}$ as its source, which means that, instead of remaining a secondary construct obtained via (2.3), the vorticity must be promoted to the status of an independent - albeit not entirely free - field in its own right. This makes it eligible to contribute directly to the variational action in such a way as to allow at a macroscopic level for the effect of a smooth distribution of microscopic quantised vortices in a realistic (compressible) model for the treatment of superfluid dynamics on a large scale, in contexts such as that of a neutron star interior, as well as ordinary laboratory applications involving liquid ${ }^{4} \mathrm{He}$, in the zero temperature limit. (To deal with the Landau type 2-constituent theory that is needed to allow for the independent entropy flux in a superfluid at finite temperature ${ }^{[8][11][15]}$, a more intricate theory would be required.)

The required form of the Lagrangian is given ${ }^{[9]}$ by

$$
\mathcal{L}=\Lambda-\frac{1}{4} \varepsilon^{\mu \nu \rho \sigma} B_{\mu \nu} w_{\rho \sigma},
$$

where the "master function", $\Lambda$, is a gauge independent scalar function of the current 3 -form $N_{\mu \nu \rho}$ (and also, of course of the background space time metric), which is specified simply by taking $\Lambda=-\rho$. The innovation to be considered here consists in allowing the master function $\Lambda$ to depend not just on $N_{\mu \nu \rho}$ but also on the vorticity 2-form $w_{\mu \nu}$. Consistently with the previous definition ${ }^{[9]}$ in the perfect fluid case, the current momentum trivector $H^{\mu \nu \rho}$ and also, in the generic case, the relevant vorticity momentum bivector $\lambda^{\mu \nu}$, are specified as partial derivatives by the (fixed background) variation rule

$$
\delta \Lambda=-\frac{1}{3 !} H^{\mu \nu \rho} \delta N_{\mu \nu \rho}-\frac{1}{2} \lambda^{\mu \nu} \delta w_{\mu \nu} .
$$

The final coupling term in (3.2) is contrived so that requiring invariance of the corresponding action integral with respect to free variations of the gauge field $B_{\mu \nu}$, for a given value of $w_{\mu \nu}$, is equivalent to imposing a field equation

$$
\nabla_{\mu} H^{\mu \nu \rho}=\frac{1}{2} \varepsilon^{\nu \rho \mu \sigma} w_{\mu \sigma} .
$$


It can be seen that this is precisely what is needed for the on shell value of $w_{\mu \nu}$ to be consistent with the original definition by the equations (2.3) and (2.4).

It remains to promulgate the rule governing the specification in (3.2) of the source field $w_{\mu \nu}$, which obviously can not be entirely free since otherwise the gauge invariance (3.1) would be violated and the system would in consequence be overdetermined. To get the desired result, the vorticity is introduced ${ }^{[9]}$ via a fibration in a manner analogous to that by which the current was introduced in the convective variational formulation ${ }^{[11]}$, but using a base space of only 2 (instead of 3) dimensions with a vorticity measure 2-form having components $w_{i j}$ with respect to local coordinates $\chi^{i}$ say for $i=1,2$, which act as a pair of freely variable (diffeomorphism gauge dependent) scalar fields. A projection $x^{\mu} \mapsto \chi^{i}$ structures the relevant spacetime neighbourhood as a fibre bundle over the material base manifold with 2 dimensional fibres interpretable as vorticity world sheets, and the base measure form $w_{i j}$ then determines the corresponding vorticity 2-form as its spacetime pullback:

$$
w_{\mu \nu}=w_{i j} \chi_{, \mu}^{i} \chi_{, \nu}^{j}
$$

It will always be possible locally to choose the coordinates to be flat with respect to the symplectic structure $w_{i j}$ on the base space, so that its non zero off diagonal components have unit magnitude, which gives the explicit expression

$$
w_{\mu \nu}=2 \chi_{,[\mu}^{1} \chi_{, \nu]}^{2} .
$$

The prescription (3.5) automatically ensures that $w_{\mu \nu}$ is closed,

$$
\nabla_{[\mu} w_{\nu \rho]}=0
$$

which is needed both as a condition for gauge invariance of the action integral and also as an integrability condition for the field equation (3.4). It also automatically ensures that $w_{\mu \nu}$ is algebraically degenerate, i.e. its determinant $|w|$ vanishes, so that it satisfies

$$
\varepsilon^{\mu \nu \rho \sigma} w_{\mu \nu} w_{\rho \sigma}=0, \quad w_{\mu \nu}=\frac{1}{2} w \varepsilon_{\mu \nu \rho \sigma} \mathcal{E}^{\rho \sigma}
$$

where $w$ is the scalar vorticity magnitude and $\mathcal{E}^{\mu \nu}$ is the unit bi-vector (as normalised by $\mathcal{E}^{\mu \nu} \mathcal{E}_{\nu \mu}=2$ ) tangential to the congruence of vortex flux 2-surfaces characterised by constant values of the pair of comoving coordinates $\chi^{i}$. The allowed variations of $w_{\mu \nu}$ are those generated by infinitesimal displacements of the form $\delta \chi^{i}=-\xi^{\mu} \partial_{\mu} \chi^{i}$, for an arbitrary spacetime vector field $\xi^{\mu}$, so they are given by the corresponding Lie derivation formula which - in view of (3.7) - takes the form

$$
\delta w_{\mu \nu}=2 \nabla_{[\mu}\left(w_{\nu] \rho} \xi^{\rho}\right) .
$$

Imposing that the action integral be invariant with respect to such variations leads finally to a basic dynamical equation given by

$$
w_{\mu \nu}\left(n^{\nu}-\nabla_{\rho} \lambda^{\rho \nu}\right)=0,
$$


which is in qualitative agreement with a form previously proposed by Lebedev and Khalatnikov ${ }^{[8]}$ on the basis of a different approach whose relationship to the present analysis will be described in the appendix.

The covariance of the foregoing formulation implies that the system will satisfy a corresponding Noether identity of the standard form

$$
\nabla_{\nu} T^{\mu \nu}=0
$$

with

$$
T^{\mu \nu}=2 \frac{\partial \mathcal{L}}{\partial g_{\mu \nu}}+\mathcal{L} g^{\mu \nu} .
$$

Working out the extra term that will be induced on the right of (3.3) by a virtual variation $\delta g_{\mu \nu}$ of the background metric, the relevant stress energy momentum density tensor is found to be given by

$$
T_{\nu}^{\mu}=\frac{1}{2} H^{\mu \rho \sigma} N_{\nu \rho \sigma}+\lambda^{\mu \sigma} w_{\nu \sigma}+\Lambda g_{\nu}^{\mu}
$$

\section{Conjugate reformulation.}

It is useful for many purposes, and in particular for relating the present approach to the previous work of Lebedev and Khalatnikov ${ }^{[8]}$, to reformulate the theory that has just been presented in a dynamically conjugate version in terms of a modified master function $\Psi$ that is obtained from the original master function $\Lambda$ by a Legendre type transformation that is derivable as follows.

We start by replacing the Kalb Ramond form $B_{\mu \nu}$ by its dual

$$
b^{\mu \nu}=\frac{1}{2} \varepsilon^{\mu \nu \rho \sigma} B_{\rho \sigma},
$$

so that by (3.1) the current vector (2.5) is expressible simply by

$$
n^{\rho}=\nabla_{\nu} b^{\rho \nu}
$$

which entails the current conservation law

$$
\nabla_{\rho} n^{\rho}=0
$$

as an immediate consequence.

Instead of postulating (3.1) or its dual version (4.2) as an axiom, we can impose it via the variational principle by introducing an appropriate Lagrange multiplier $\pi_{\rho}$ and replacing the original Lagrangian $\mathcal{L}$ as given by (3.2) by the correspondingly augmented Lagrangian, whose form, after subtraction of a divergence which does not affect the field equations, is given by

$$
\mathcal{L}^{\dagger}=\mathcal{L}+\pi_{\rho}\left(\nabla_{\nu} b^{\rho \nu}-n^{\rho}\right)-\nabla_{\nu}\left(\pi_{\rho} b^{\rho \nu}\right),
$$

in which $n^{\rho}$ and $\pi_{\rho}$ as well as $b^{\rho \sigma}$ are to be considered as independent free variables, while as before $w_{\rho \sigma}$ is also to be considered as independent though not entirely free but given by (3.5) 
which means that it is constrained to vary according to (3.9). Invariance of the ensuing action with respect to variation of the current $n^{\mu}$ itself gives the dynamical relation

$$
\pi_{\rho}=\mu_{\rho}
$$

from which the requirement of invariance with respect to the other allowed variations can be seen to give back the same dynamical equations as were given by the original Lagrangian $\mathcal{L}$.

Instead of considering the trivial relation (4.5) as a dynamical relation, let us now simply impose it as a defining restriction, so that the new Lagrangian (4.4) will reduce to the form

$$
\mathcal{L}^{\dagger}=\Psi+b^{\rho \sigma}\left(\nabla_{\rho} \mu_{\sigma}-\frac{1}{2} w_{\rho \sigma}\right)
$$

where

$$
\Psi=\Lambda-\mu_{\rho} n^{\rho} .
$$

It can be seen that the (fixed background) variation of this function $\Psi$ will be simply expressible as

$$
\delta \Psi=-n^{\rho} \delta \mu_{\rho}-\frac{1}{2} \lambda^{\rho \sigma} \delta w_{\rho \sigma},
$$

which suggests that instead of regarding the components $n^{\rho}$ or equivalently $N_{\mu \nu \rho}$ as independent field variables from which the components $\mu_{\rho}$ or equivalently $H^{\mu \nu \rho}$ are derived by partial differentiation according to (3.3), it would be convenient to treat the components $\mu_{\rho}$ as independent variables, from which the components $n^{\rho}$ are obtained by partial differentiation according to (4.8).

This Legendre type transformation provides a new formulation in which the dual bivector $b^{\rho \sigma}$ to the Kalb Ramond form comes in as a Lagrange multiplier imposing the condition that the vorticity 2 -form should be the exterior derivative of the momentum 1-form. In this conjugate reformulation, $\Psi$ is to be considered as a function just of the freely variable momentum covector $\mu_{\rho}$ and of the independent but constrained vorticity 2 -form $w_{\rho \sigma}$ whose variation is governed by (3.9). Under these conditions the corresponding variation of the new Lagrangian (4.6) will be given by

$$
\delta \mathcal{L}^{\dagger}-\nabla_{\rho}\left(b^{\rho \sigma} \delta \mu_{\sigma}\right)=\left(\nabla_{\sigma} b^{\rho \sigma}-n^{\rho}\right) \delta \mu_{\rho}+\left(\nabla_{\rho} \mu_{\sigma}-\frac{1}{2} w_{\rho \sigma}\right) \delta b^{\rho \sigma}-\frac{1}{2}\left(b^{\rho \sigma}+\lambda^{\rho \sigma}\right) \delta w_{\rho \sigma} .
$$

As well as giving back the relation (4.2) and the specification (2.3) of the vorticity as the exterior derivative of the momentum, the use of (3.9) in the new variational principle gives back the main dynamical relation (3.10) in the form

$$
j^{\rho} w_{\rho \sigma}=0
$$

where the modified current vector is given by the definition

$$
j^{\rho}=\nabla_{\sigma}\left(b^{\rho \sigma}+\lambda^{\rho \sigma}\right)=n^{\rho}-\nabla_{\sigma} \lambda^{\sigma \rho},
$$

from which it is apparent that, like the particle current vector $n^{\mu}$ itself, the modified current is also conserved:

$$
\nabla_{\rho} j^{\rho}=0 .
$$


Since the contribution to the action from the extra terms introduced in the transformation (4.4) does not depend on the metric, it follows that the stress momentum energy tensor derived from the modified Lagrangian $\mathcal{L}^{\dagger}$, namely

$$
T_{\sigma}^{\rho}=n^{\rho} \mu_{\sigma}-\lambda^{\rho \nu} w_{\nu \sigma}+\Psi g_{\sigma}^{\rho}
$$

will automatically be the same as the tensor (3.13) that was obtained from the original Lagrangian $\mathcal{L}$.

\section{Explicit development.}

The form of the master function $\Lambda$ for the original formulation given in Section 3 can be made more explicit by expressing it in terms of the three independent scalars that can be constructed from the tensors $n^{\rho}$ and $w_{\rho \sigma}$ on which it depends. These can be conveniently be taken to be the magnitude $n$ of the particle current vector $n^{\rho}$ itself, the scalar magnitude $w$ of the vorticity covector $w_{\rho \sigma}$ and the magnitude $\zeta$ of the associated Joukowsky lift force density vector $\zeta_{\rho}$ as defined by

$$
n^{2}=-n^{\rho} n_{\rho}, \quad w^{2}=\frac{1}{2} w_{\rho \sigma} w^{\rho \sigma}, \quad \zeta^{2}=\zeta_{\rho} \zeta^{\rho},
$$

where the Joukowsky vector is defined as

$$
\zeta_{\rho}=w_{\rho \sigma} n^{\sigma}=\frac{1}{2} w \mathcal{E}^{\mu \nu} N_{\mu \nu \rho}
$$

This vector is interpretable as representing the volume density of force that would be exerted on the vortices as an expression of the Magnus effect, by the relative flux (if any) of the fluid according to the simple formula originally derived by Joukowsky (or Zhukovski, depending on how one transliterates from Cyrillic) for flow past a long aerofoil. The coefficients appearing in the derivation of $\Lambda$ can be expressed explicitly in terms of partial derivatives with respect to these scalars in the form

$$
\begin{aligned}
\frac{1}{2} H^{\mu \nu \rho} & =-\frac{\partial \Lambda}{\partial n^{2}} N^{\mu \nu \rho}-3 \frac{\partial \Lambda}{\partial \zeta^{2}} w \mathcal{E}^{[\mu \nu} \zeta^{\rho]}, \\
\lambda^{\rho \sigma} & =-2 \frac{\partial \Lambda}{\partial w^{2}} w^{\rho \sigma}-4 \frac{\partial \Lambda}{\partial \zeta^{2}} \zeta^{[\rho} n^{\sigma]} .
\end{aligned}
$$

The stress energy momentum tensor can thereby be rewritten in the manifestly symmetric form

$$
T_{\nu}^{\mu}=-\frac{\partial \Lambda}{\partial n^{2}} N^{\mu \sigma \tau} N_{\nu \sigma \tau}-2 \frac{\partial \Lambda}{\partial w^{2}} w^{\mu \rho} w_{\nu \rho}-2 \frac{\partial \Lambda}{\partial \zeta^{2}} \zeta^{\mu} \zeta_{\nu}+\left(\Lambda-2 \frac{\partial \Lambda}{\partial \zeta^{2}} \zeta^{2}\right) g_{\nu}^{\mu}
$$

An analogously explicit analysis of the conjugate formulation developed in Section 4 can be made by expressing the generalised pressure function $\Psi$ in terms of the three independent scalars that can be constructed from the tensors $\mu_{\rho}$ and $w_{\rho \sigma}$ on which it depends. These can be taken to be the magnitude $w$ of the vorticity vector, as already given by (3.8) or (5.1) together 
with the magnitude $\mu$ of the momentum covector and the magnitude $h$ of the helicity vector as given by

$$
\mu^{2}=-\mu_{\rho} \mu^{\rho}, \quad h^{2}=h^{\rho} h_{\rho},
$$

where the helicity vector ${ }^{[16]}$ is given by the definition

$$
h^{\mu}=\frac{1}{2} \varepsilon^{\mu \nu \rho \sigma} \mu_{\nu} w_{\rho \sigma}=w \mu_{\rho} \mathcal{E}^{\rho \mu},
$$

which, by the degeneracy property (3.6), is such as to ensure that it will be conserved:

$$
\nabla_{\mu} h^{\mu}=0 .
$$

In terms of partial derivatives with respect to $w^{2}, \mu^{2}$ and $h^{2}$ one obtains the expressions

$$
\begin{gathered}
n^{\rho}=2 \frac{\partial \Psi}{\partial \mu^{2}} \mu^{\rho}+2 \frac{\partial \Psi}{\partial h^{2}} w h_{\sigma} \mathcal{E}^{\sigma \rho}, \\
\lambda^{\rho \sigma}=-2 \frac{\partial \Psi}{\partial w^{2}} w^{\rho \sigma}-2 \frac{\partial \Psi}{\partial h^{2}} h_{\nu} H^{\nu \rho \sigma} .
\end{gathered}
$$

The corresponding manifestly symmetric expression for the stress momentum energy density tensor (4.13) is found to have the form

$$
T_{\sigma}^{\rho}=2 \frac{\partial \Psi}{\partial w^{2}} w^{\rho \tau} w_{\tau \sigma}+2 \frac{\partial \Psi}{\partial \mu^{2}} \mu^{\rho} \mu_{\sigma}+2 \frac{\partial \Psi}{\partial h^{2}} h^{\rho} h_{\sigma}+\left(\Psi-2 \frac{\partial \Psi}{\partial h^{2}} h^{2}\right) g_{\sigma}^{\rho} .
$$

\section{Deviations from Perfect Fluid Limit.}

Not only in the usual terrestrial laboratory experiments but also in the neutron star applications for which the present theory is principally intended, the relevant macroscopic angular velocities are sufficiently small - and the ensuing microscopic vortex lines therefore sufficiently widely separated - for it to be a good approximation to consider the effect of the associated vorticity as a small perturbation to the dynamics. It will therefore be useful to formulate the theory in terms of deviations from a simple barytropic fluid model governed by an equation of state giving the zero vorticity density $\rho$ just as a function of the particle number density $n$ or equivalently, in conjugate, form giving the zero vorticity pressure $P$ as a function of the effective mass per particle $\mu$.

As a premilinary step, to prepare the way for a perturbation analysis in which the deviations will be considered to be small, we shall start without any loss of generality by considering deviations of unrestricted amplitude, simply decomposing the master function $\Lambda$ as the sum of the perfect fluid contribution, $\rho_{\diamond}$ say, to which it reduces when the vorticity is zero, together with a vorticity dependent deviation term $\Lambda$ in the form

$$
\Lambda=-\rho_{\oslash}+\Lambda^{\prime}, \quad \rho_{\oslash}=\rho\{n\} .
$$


We can also simply decompose the conjugate master function $\Psi$ in the analogous manner as the sum of the perfect fluid contribution, $P_{\ominus}$ say, to which it reduces when the vorticity is zero, together with a vorticity dependent deviation term $\dot{\Psi}$ in the form

$$
\Psi=P_{\ominus}+\Psi^{`}, \quad P_{\ominus}=P\{\mu\} .
$$

With respect to the vorticity tensor $w_{\rho \sigma}$ itself, the partial derivatives of these deviation terms will be given by the same tensor $\lambda^{\rho \sigma}$ as for the total, but they will determine a reduced momentum covector $\mu_{\rho}^{\prime}$, and a reduced current vector $n^{\prime \rho}$ according to the specifications

$$
\begin{gathered}
\delta \Lambda^{\prime}=\mu_{\rho}^{\prime} \delta n^{\rho}-\frac{1}{2} \lambda^{\rho \sigma} \delta w_{\rho \sigma}, \\
\delta \Psi^{`}=-n^{`} \delta \mu_{\rho}-\frac{1}{2} \lambda^{\rho \sigma} \delta w_{\rho \sigma} .
\end{gathered}
$$

Defining the particle current reference state unit vector $u_{\odot}^{\rho}$ and the corresponding perfect fluid momentum contribution $\mu_{\odot \rho}$ by

$$
n^{\rho}=n u_{\oslash}^{\rho}, \quad \mu_{\oslash \rho}=\mu_{\oslash} u_{\oslash \rho}, \quad \mu_{\oslash}=\mu\{n\}=\frac{d \rho_{\odot}}{d n}
$$

one obtains the total momentum contribution from (6.1) in the form

$$
\mu_{\rho}=\mu_{\oslash}+\mu_{\rho}^{\prime} .
$$

Similarly defining the momentum reference state unit vector $u_{\ominus}^{\rho}$ and the corresponding perfect fluid particle current contribution $n_{\ominus}^{\rho}$ by

$$
\mu_{\rho}=\mu u_{\ominus}, \quad n_{\ominus}^{\rho}=n_{\ominus} u_{\ominus}^{\rho}, \quad n_{\ominus}=n\{\mu\}=\frac{d P_{\ominus}}{d \mu},
$$

one obtains the total particle current contribution from (6.2) in the form

$$
n^{\rho}=n_{\oslash}^{\rho}+n^{\curlywedge}
$$

Defining the particle current reference state perfect fluid contribution by

$$
T_{\oslash}^{\rho \sigma}=\rho_{\oslash} u_{\oslash}^{\rho} u_{\oslash}^{\sigma}+P_{\oslash}\left(g^{\rho \sigma}+u_{\oslash}^{\rho} u_{\oslash}^{\sigma}\right), \quad P_{\oslash}=n \mu_{\oslash}-\rho_{\oslash},
$$

one can express the total stress momentum energy density contribution in the form

$$
T_{\sigma}^{\rho}=T_{\oslash \sigma}^{\rho}+T_{\sigma}^{\rho}
$$

with

$$
T_{\sigma}^{\rho}=n^{\rho} \mu_{\sigma}^{\prime}+\lambda^{\rho \nu} w_{\sigma \nu}+\left(\Lambda^{\prime}-n^{\nu} \mu_{\nu}^{\prime}\right) g_{\sigma}^{\rho} .
$$

This can be interpreted as the stress momentum energy density contribution of a generalised kind of Stachel-Letelier type ${ }^{[17][18][19]}$ "string fluid", given by the vorticity dependent action contribution $\Lambda^{\prime}$. 
To obtain the analogous contribution from $\Psi^{`}$ in the conjugate formulation we similarly define the momentum reference state perfect fluid contribution by

$$
T_{\ominus}^{\rho \sigma}=\rho_{\ominus} u_{\ominus}^{\rho} u_{\ominus}^{\sigma}+P_{\ominus}\left(g^{\rho \sigma}+u_{\ominus}^{\rho} u_{\ominus}^{\sigma}\right), \quad \rho_{\ominus}=n \mu_{\ominus}-P_{\ominus} .
$$

We can then express the total stress momentum energy density contribution in the alternative form

$$
T_{\sigma}^{\rho}=T_{\ominus \sigma}^{\rho}+T_{\sigma}^{\wedge}
$$

with

$$
T^{{ }^{\rho}}{ }_{\sigma}=n^{` \rho} \mu_{\sigma}+\lambda^{\rho \nu} w_{\sigma \nu}+\Psi g^{\rho}{ }_{\sigma} .
$$
by

It can be seen from (4.7) that the mutually conjugate deviation contributions will be related

$$
\Lambda^{\prime}-\Psi^{`}=P_{\ominus}-P_{\oslash}+n^{\nu} \mu_{\nu}^{\prime}=\rho_{\oslash}-\rho_{\ominus}+n^{\iota \nu} \mu_{\nu} .
$$

In the limit as deviations from the zero vorticity state tend to zero, it can be seen from the defining relations (2.1) that we shall have

$$
P_{\ominus}-P_{\ominus} \sim n\left(\mu-\mu_{\ominus}\right), \quad \rho_{\oslash}-\rho_{\ominus} \sim \mu\left(n-n_{\ominus}\right) .
$$

Since by (6.6) and (6.8) we shall have

$$
\mu_{\odot}-\mu \sim u_{\ominus}^{\rho} \mu_{\rho}^{\prime}, \quad n_{\ominus}-n \sim u_{\ominus \rho} n^{\iota^{\rho}},
$$

it can be seen from (6.15) that in this limit the deviations of the two mutually conjugate kinds of master function will coincide, i.e. we shall have

$$
\Psi^{`} \sim \Lambda^{\prime} .
$$

\section{Asymptotically Separable Model for the Weak Vortic- ity Limit.}

A further simplification can be obtained by postulating that the master function has a separable form such that the vorticity dependence in the deviation term is contained in a negative factor, $-\Upsilon$ say, depending only on the scalar magnitude $w$, with a positive coefficient $\Phi^{2}$ that depends only on the relevant perfect fluid reference state: this state will be labelled by $\oslash$ when determined by the particle current magnitude $n$ so that the deviation $\Lambda^{\prime}$ defined in (6.1) will have the form $\Lambda^{\prime}=-\Phi_{\odot}^{2} \Upsilon$; alternatively the reference state will be labelled by $\ominus$ when determined by the effective mass per particle $\mu$ so that the corresponding deviation defined by (6.2) will have the form $\Psi^{`}=-\Phi_{\ominus}^{2} \Upsilon$. It must be noticed that, although in general a model that is separable in the particle number representation (6.1) will not be exactly separable in the corresponding chemical potential representation (6.2) and conversely, it can be seen from the work of the preceeding section that the property of separability is nevertheless conserved 
by the Legendre transformation in the weak vorticity limit. Such an asymptotically separable form, as given by

$$
\Lambda^{\prime} \sim-\Phi_{\ominus}^{2} \Upsilon\{w\}, \quad \Psi^{`} \sim-\Phi_{\ominus}^{2} \Upsilon\{w\},
$$

in the relevant weak vorticity limit characterised by $\Upsilon \rightarrow 0$, is suggested by our recent analysis ${ }^{[11]}$ of the average stress momentum energy density for an individual vortex cell. The results of this analysis can be matched in a very satisfactory manner by an appropriate choice of the single variable functions $\Upsilon$ and $\Phi$.

Dropping the label $\oslash$ or $\ominus$ in asymptotic formulae since there is no need to distinguish between the two kinds of reference state because either interpretation would be valid, as illustrated by the relation $\Phi_{\diamond} \sim \Phi \sim \Phi_{\ominus}$, we can express the ensuing asymptotic forms of the particle current and momentum deviations given by (6.3) and (6.4) in the form

$$
\mu_{\rho}^{\prime} \sim \frac{\Upsilon}{\mu} \frac{d \Phi^{2}}{d n} \mu_{\rho}, \quad n^{` \rho} \sim-\frac{\Upsilon}{n} \frac{d \Phi^{2}}{d \mu} n^{\rho},
$$

and we similarly obtain

$$
\lambda^{\mu \nu} \sim \frac{1}{2} \lambda \varepsilon^{\mu \nu \rho \sigma} \mathcal{E}_{\rho \sigma}, \quad \lambda \sim \Phi^{2} \frac{d \Upsilon}{d w} .
$$

On substituting this in the expression (6.11) for the deviation of the stress momentum energy tensor from that of the particle current reference state, due to the vorticity dependent action contribution $\Lambda^{\prime}$, we see that the asymptotic form of this deviation will be expressible in terms of the fundamental tangential and normal projection tensors associated with the flux 2 -surfaces ${ }^{[20][21]}$, as given respectively by

$$
\eta^{\rho}{ }_{\sigma}=\mathcal{E}^{\rho}{ }_{\nu} \mathcal{E}^{\nu}{ }_{\sigma}
$$

and

$$
\perp_{\sigma}^{\rho}=g_{\sigma}^{\rho}-\eta_{\sigma}^{\rho},
$$

in the form

$$
T_{\sigma}^{\rho} \sim n \frac{d \Phi^{2}}{d n} \Upsilon u^{\rho} u_{\sigma}-\left(\Phi^{2}-n \frac{d \Phi^{2}}{d n}\right) \Upsilon \eta_{\sigma}^{\rho}+\Pi^{\prime} \perp_{\sigma}^{\rho},
$$

in which, relative to the particle current reference state, the relevant effective vorticity pressure $\Pi^{\prime}$, acting transversely to the vortex flux lines, will be given by

$$
\Pi^{\prime} \sim w \lambda-\left(\Phi^{2}-n \frac{d \Phi^{2}}{d n}\right) \Upsilon .
$$

In the equivalent conjugate formulation, the contribution from $\Psi^{`}$, giving the deviation of the stress momentum energy density tensor with respect to that of the particle momentum reference state, will be analogously expressible in the form

$$
T_{\sigma}^{{ }_{\sigma}} \sim-\mu \frac{d \Phi^{2}}{d \mu} \Upsilon u^{\rho} u_{\sigma}-\Phi^{2} \Upsilon \eta_{\sigma}^{\rho}+\Pi^{{ }^{\prime}} \perp_{\sigma}^{\rho},
$$


in which, relative to the particle momentum reference state, the effective vorticity pressure $\Pi^{\prime}$, relative to the momentum reference state, will be given by

$$
\Pi \sim w \lambda-\Phi^{2} \Upsilon
$$

In order to get the formulae (7.6), (7.7), (7.8) and (7.9) to agree with the corresponding formulae as obtained in the preceeding work ${ }^{[15]}$ by averaging over an individual vortex cell with circulation given by the standard quantum unit $\kappa=2 \pi \hbar$, it suffices to take the functions $\Phi$ and $\Upsilon$ to be given by

$$
\Phi^{2}=\frac{n}{\mu}, \quad \Upsilon=\frac{\hbar}{4} w \ln \left\{\frac{w_{\odot}}{w}\right\},
$$

where $w_{\odot}$ is a fixed cut off vorticity value - whose exact value is unimportant in the limit $w<<w_{\odot}$ under consideration. The specification (7.10) means that the function $\Phi$ is to be identified with the dilation amplitude that has been shown to have a specially important role in the newly developed variational formulation of perfect fluid theory ${ }^{[9]}$. The logarithmic derivative of this function is expressible in terms of the corresponding sound speed (2.2) according to either of the equivalent mutually conjugate specifications

$$
\frac{n}{\Phi^{2}} \frac{d \Phi^{2}}{d n}=1-c_{\mathrm{S}}^{2}, \quad \frac{\mu}{\Phi^{2}} \frac{d \Phi^{2}}{d \mu}=c_{\mathrm{S}}^{-2}-1 .
$$

Since (7.10) also implies

$$
\lambda w \sim \Phi^{2} \Upsilon
$$

the deviation with respect to the particle current reference state is thus obtainable from (7.6) and (7.7) in the form

$$
T_{\sigma}^{\rho} \sim \Phi^{2} \Upsilon\left(\left(1-c_{\mathrm{S}}^{2}\right) u^{\rho} u_{\sigma}-c_{\mathrm{S}}^{2} \eta_{\sigma}^{\rho}+\left(1-c_{\mathrm{S}}^{2}\right) \perp_{\sigma}^{\rho}\right)
$$

while the conjugate formula for the deviation with respect to the particle momentum reference state is similarly obtainable from (7.8) and (7.9) in the form

$$
T_{\sigma}^{\rho_{\sigma}} \sim \Phi^{2} \Upsilon\left(\left(1-c_{\mathrm{S}}^{-2}\right) u^{\rho} u_{\sigma}-\eta_{\sigma}^{\rho}\right), \quad \Pi \sim 0
$$

The upshot of the foregoing analysis is that a master function given in terms of functions of the form (7.10) by

$$
\Lambda=-\rho_{\oslash}-\Phi_{\oslash}^{2} \Upsilon\{w\}+o\{\Upsilon\}
$$

(where the label $\oslash$ indicates that the quantities concerned are to be considered as functions just of the particle number density $n$ ) provides a vortex fibration model whose stress momentum energy density tensor is consistent with what has been derived ${ }^{[15]}$ by averaging that of an individual vortex cell in the weak vorticity limit.

So long as $w$ varies within a few orders of magnitude of some mean value $\langle w\rangle$ that is itself a great many orders of magnitude smaller than the the cut off value $w_{\odot}$ appearing in (7.10), i.e.

$$
w \approx\langle w\rangle, \quad\langle w\rangle<<w_{\odot}
$$


which will be a very good approximation in typical contexts that can be envisaged for the application of the present theory, and in particular in the case of the neutron star matter for which it is principally intended, then the variation of the logarithmic factor in the formula (7.10) will be negligible, so that it will suffice to replace it simply by a linear function, with a constant coefficient $\mathcal{K}$ proportional to the circulation $\kappa$ round an individual vortex, that will be given by

$$
\Upsilon=\mathcal{K} w, \quad \mathcal{K}=\frac{\kappa}{8 \pi} \ln \left\{\frac{w_{\odot}}{\langle w\rangle}\right\}=\frac{\hbar}{4} \ln \left\{\frac{w_{\odot}}{\langle w\rangle}\right\}
$$

\section{Dilatonic model.}

As an ansatz for matching the available results obtained ${ }^{[15]}$ from the analysis of individual vortex cells, the asymptotic form (7.15) obtained in the preceeding section is not the only possibility, but it is the the only one that satisfies the simplifying condition that the master function $\Lambda$ be dependent only on the first two scalars, $n$ and $w$, of the triplet introduced in (5.1), or equivalently that the conjugate master function $\Psi$ depend only on $w$ and the first scalar, $\mu$, of the pair introduced in (5.6):

$$
\frac{\partial \Lambda}{\partial \zeta}=0 \quad \Leftrightarrow \quad \frac{\partial \Psi}{\partial h}=0 .
$$

Unlike an alternative simplifying assumption implicit in the earlier work of Lebedev and Khalatnikov[8] which will be described in the appendix, the condition (8.1) has the convenient feature of treating the mutually conjugate functions $\Lambda$ and $\Psi$ on the same footing. The extent to which such a simplifying condition is accurate in the weak vorticity limit with which we are concerned remains to be checked by future work on non-axisymmetric vortex cells, but until there is any evidence that a more complicated ansatz may be needed, the most reasonable procedure is to adopt what is obviously the simplest provisionally admissible supposition, namely (8.1), as a tentative working hypothesis.

Proceeding on this basis, which means that the general laws (3.3) and (4.8) will simplify just to

$$
\delta \Lambda=-\mu \delta n-\lambda \delta w
$$

and

$$
\delta \Psi=n \delta \mu-\lambda \delta w
$$

with

$$
\Psi=\Lambda+n \mu,
$$

the relations $(5.3),(5.4)$ or their conjugate forms (5.9), (5.10), will reduce just to

$$
\lambda^{\rho \sigma}=\frac{\lambda}{w} w^{\rho \sigma}
$$

and

$$
\mu^{\rho}=\Phi^{-2} n^{\rho}, \quad \Phi^{-2}=\frac{\mu}{n}
$$


so that the expression (4.13) for the stress momentum energy density tensor will reduce simply to

$$
T_{\sigma}^{\rho}=\Phi^{-2} n^{\rho} n_{\sigma}-\lambda w^{-1} w^{\rho \nu} w_{\nu \sigma}+\Psi g_{\sigma}^{\rho} .
$$

As a compromise between the alternative mutually conjugate master functions $\Lambda$ and $\Psi$, it is particularly convenient in such a case to work with a potential energy function $\mathcal{U}$ defined by

$$
\mathcal{U}=-\frac{1}{2}(\Lambda+\Psi)
$$

whose variation law, subject to (8.1), is obtainable from (8.2) and (8.3) in the form

$$
\delta \mathcal{U}=\lambda \delta w+\frac{\mu n}{\Phi} \delta \Phi .
$$

This shows that - except in the degenerate "stiff" case for which $\mu$ is just proportional to $n$ so that $\Phi$ is constant and $\delta \Phi$ vanishes - the potential $\mathcal{U}$ can be used as a master function whose specification in terms of the independent variables $w$ and $\Phi$ determines the original master function $\Lambda$ and its conjugate $\Psi$ by the formulae

$$
\Lambda=-\frac{n^{2}}{2} \Phi^{-2}-\mathcal{U}, \quad \Psi=\frac{\mu^{2}}{2} \Phi^{2}-\mathcal{U}
$$

where the quantities $n^{2}$ and $\mu^{2}$ are obtained as functions of the vorticity amplitude $w$ and the "dilatonic" amplitude $\Phi$ via the partial differential relations

$$
\mu^{2}=\Phi^{-1} \frac{\partial \mathcal{U}}{\partial \Phi}, \quad n^{2}=\Phi^{3} \frac{\partial \mathcal{U}}{\partial \Phi}
$$

Subject to the assumption (8.1) the potential defined by (8.9) can be used to rewrite the Lagrangian (3.2) in the form

$$
\mathcal{L}=-\frac{1}{2} \Phi^{-2} n^{2}-b^{\rho \sigma} \chi_{, \rho}^{1} \chi_{, \sigma}^{2}-\mathcal{U}\{\Phi, w\}
$$

which can be used as the basis of a new variational formulation in which the independent field variables are classifiable in three subsets: the first consists of the dual Kalb Ramond bivector components $b^{\rho \sigma}$, as defined by (4.1) or (4.2), in terms of which the current amplitude $n$ is defined by

$$
n^{2}=-\left(\nabla_{\rho} b_{\nu}{ }^{\rho}\right) \nabla_{\sigma} b^{\nu \sigma}
$$

the second subset consists of the vorticity base coordinates $\chi^{1}$ and $\chi^{2}$ as defined by (3.5), in terms of which, with respect to a flat symplectic gauge (3.6), the vorticity amplitude will be given by

$$
w^{2}=2 \chi_{,[\mu}^{1} \chi_{, \nu]}^{2}\left(\nabla^{\mu} \chi^{1}\right) \nabla^{\nu} \chi^{2}
$$

finally the third subset consists just of the dilatonic amplitude $\Phi$ which is to be considered here as an independently variable scalar in its own right - except in the degenerate "stiff" case, for which $\mathcal{U}$ depends only on $w$ with $\Phi$ acting merely as a fixed coupling constant. 
An attractive feature of the "dilatonic" formulation set up in this way is that it allows the physically required asymptotic form (7.15) to be matched by taking the new master function $\mathcal{U}$ to have a form that is not just separable but more specifically linearly separable with respect to $\Phi^{2}$, meaning that it is expressible as

$$
\mathcal{U}\{\Phi, w\}=V\{\Phi\}+\Phi^{2} \Upsilon\{w\}
$$

for suitable single variable functions $V$ of $\Phi$ and $\Upsilon$ of $w$. The condition of matching (7.15) in the weak vorticity limit leaves no freedom of choice in the specification of these single variable functions: $\Upsilon$ here must be the same as the function that is denoted by the same symbol in (7.15) and that is given explicitly by (7.10) or with sufficient precision for practical purposes by (7.17), while $V$ must be the same as the function denoted by the same symbol in a recent discussion of the dilatonic formulation of the perfect fluid limit ${ }^{[9]}$, which means that it is determined by the equation of state for the mass density $\rho$ of the underlying perfect fluid as a function of its conserved number density $n$ according to the parametric specification

$$
V\{n\}=\rho\{n\}-\frac{1}{2} n \frac{d \rho\{n\}}{d n}, \quad \Phi^{-2}=n^{-1} \frac{d \rho\{n\}}{d n} .
$$

The exceptional Zel'dovich ${ }^{[10]}$ case characterised by a fixed dilatonic amplitude $\Phi=a$ say arises from an equation of state of the "stiff" type $\rho=n^{2} / 2 a^{2}+b$ where $b$ is also fixed (acting just as a cosmological constant) which gives $V=b$ and hence $\mathcal{U}=b+a^{2} \Upsilon$.

In any such linearly separable model the vorticity coefficient $\lambda$ will be given just by

$$
\lambda=\Phi^{2} \frac{d \Upsilon}{d w}
$$

and, except in the degenerate "stiff" case (for which $d V / d \Phi$ is indeterminate), the original master function will have a value that is given by a linearly separable expression of its own, namely

$$
\Lambda=-\frac{\Phi}{2} \frac{d V}{d \Phi}-V-2 \Phi^{2} \Upsilon
$$

while the conjugate function $\Psi$ turns out to have a corresponding expression in which the dependence on $w$ drops out altogether, leaving just

$$
\Psi=\frac{\Phi}{2} \frac{d V}{d \Phi}-V
$$

In applications for which the linear formula (7.17) for the vorticity dependent factor $\Upsilon$ is considered to be sufficiently accurate, so that (8.17) will take the specific form

$$
\lambda=\mathcal{K} \Phi^{2}
$$

while (8.11) will take the specific form

$$
n^{2}=\Phi^{3} \frac{d V}{d \Phi}+2 \mathcal{K} \Phi^{4} w
$$


the Lagrangian (8.12) of the dilatonic formulation can be written out with a fully explicit presentation of the gradient dependence, which in this case will be homogeneously quadratic, as

$$
\mathcal{L}=\frac{1}{2 \Phi^{2}}\left(\nabla_{\rho} b_{\nu}{ }^{\rho}\right) \nabla_{\sigma} b^{\nu \sigma}-b^{\rho \sigma} \chi_{, \rho}^{1} \chi_{, \sigma}^{2}-\mathcal{K} \Phi^{2} \sqrt{2 \chi_{,[\mu}^{1} \chi_{, \nu]}^{2}\left(\nabla^{\mu} \chi^{1}\right) \nabla^{\nu} \chi^{2}}-V\{\Phi\}
$$

In the application of the corresponding action principle, variation with respect to the dual Kalb Ramond bivector components $b^{\rho \sigma}$ gives the formula (2.3) for the vorticity form (3.6) in terms of the momentum covector given by (8.6) in conjunction with the definition (4.2); variation with respect to the vorticity base coordinates $\chi^{1}$ and $\chi^{2}$ with the definition (8.5) gives the basic dynamical equation of motion (3.10); while finally - except in the "stiff" case for which $\Phi$ is simply fixed as a coupling constant - variation with respect to the dilatonic amplitude itself gives the equation (8.21) whose solution (for a given form of the equation of state function $V\{\Phi\}$ ) determines the value of this amplitude $\Phi$ as a function of the current magnitude $n$ given by $(8.13)$.

In simple cases the correspondence (8.16) between the equation of state for $\rho$ as a function of $n$ and the associated equation of state for $V$ as a function of $\Phi$ can be made explicit as discussed in the recent analysis of the perfect fluid case ${ }^{[9]}$. The simplest example of relevance as an approximation for neutron star matter is that of the standard relativistic polytrope with index $\gamma=4 / 3$ as given, for a constant coefficient $a$, by

$$
\rho=\frac{3 a}{2} n^{4 / 3} \quad \Rightarrow \quad V=2 a^{3} \Phi^{4} .
$$

It is not to be expected that such a simple model could provide a completely accurate description for the most general kinds of astrophysical and terrestrial applications. High accuracy would require an even more elaborate treatment allowing for the effect ${ }^{[2]}$ of vortex lattice rigidity, and for many purposes in the context of neutron star applications it will also be necessary to allow for the further complication of magnetic effects involving the independently conducting proton fluid component ${ }^{[1][2]}$. However the most serious limitation of the above model may turn out to be due to inadequate allowance for the effect of relative flow between the averaged particle current and the vorticity surfaces. Such an effect was deliberately excluded from consideration in the preceeding microscopic analysis ${ }^{[15]}$ of an individual vortex cell that provided the averages to which the macroscopic model proposed here has been matched. A more difficult microscopic analysis (lacking the cylindrical symmetry that facilitated the previous calculation) still needs to be carried out by future work to determine how the relevant averages are affected by the inclusion of the effect of relative flow. Until the results of such a more complete microscopic analysis are available, the simple model set up in the present and preceeding section should be considered to be physically trustworthy only in the limit of negligibly small values of the Joukowsky vector (5.2) which can be considered as specifying the magnitude and direction of the relative flow.

We may sum up by saying that within the subcategory characterised by the ansatz (8.1) postulating that the master function $\Lambda$ depends only on the current magnitude $n$ and the vorticity scalar $w$ but not on the Joukowsky scalar $\zeta$, the condition of matching the results of our preliminary microscopic analysis ${ }^{[15]}$ for the case $\zeta=0$ leads uniquely, as far as the weak vorticity limit is concerned, to a model of the kind set up in Section 7 and Section 8. A more 
complete microscopic analysis may show that within the general framework provided in Section 3 some other ansatz, such as the example ${ }^{[8]}$ described in Appendix B, whereby $\Lambda$ is made to depend also on $\zeta$, will be necessary for matching cases for which $\zeta$ differs significantly from zero. Nevertheless the obvious ansatz of $\zeta$ independence has provided an elegant prototype model, which should provide a reasonably accurate description of stationary configurations in which there is no relative flow of the fluid relative to the vortex congruence, and perhaps even a moderately realistic description under more general circumstances. Considered as a toy, this prototype model can also be used, as described in Appendix A, to provide a more general illustration of the application of cosmic string theoretical concepts to superfluid dynamics that was considered by Davis and Shellard ${ }^{[4][5]}$ within the restrictive framework of the "stiff" limit characterised by a fixed value of $\Phi$ with vanishing $V$.

\section{Acknowledgement.}

The authors thank G.L. Comer, R.L. Davis, I.M. Khalatnikov, L. Lindblom, X. Martin, P. Peter, D. Priou, J. Shaham, and E.P.S. Shellard for instructive discussions.

\section{Appendix A: The tie up with cosmic string theory.}

The preceeding theory is designed to represent the macroscopically averaged effect of a congruence of microscopic vortices of which each individual member can be considered as an example of the kind of topological defect known in a cosmological context as a global cosmic string, of which the simplest type is provided by the axion field theory in which the relevant underlying fluid model is of the "stiff" kind in which the dilatonic amplitude $\Phi$ has a constant value. (The qualification "global" is needed here to distinguish such a relatively extended configuration from the more strictly string-like gauge coupled case for which, instead of being logarithmically divergent, the defect energy distribution is locally confined: such "local" string defects occur in the familiar laboratory context of ordinary metallic superconductors, and are also predicted - in coexistence with the dynamically dominant global string defects with which

the present work is concerned - in the interior of neutron $\operatorname{stars}^{[1][2]}$, where the relevant gauge coupled superconducting current is constituted from protons.)

To see how the theory set up in Section 8 is to be interpreted in the terms of the technical machinery originally developed to describe string defects of the cosmic type, it is to be remarked that the general form (8.7) that is obtained from (8.1) for the stress momentum energy density tensor is decomposable in a natural manner as the sum,

$$
T_{\sigma}^{\rho}=T_{\circ \sigma}^{\rho}-\frac{w}{\kappa} T \eta_{\sigma}^{\rho}
$$

of a spacially isotropic (non-barytropic) perfect fluid type contribution

$$
T_{\mathrm{o} \sigma}^{\rho}=n^{\rho} \mu_{\sigma}+(\Psi+\lambda w) g_{\sigma}^{\rho}
$$

together with a term of the form that would be given by a Stachel-Letelier ${ }^{[17][18][19]}$ type "string fluid", whereby each individual vortex cell contributes as if it were a string of the simple 
degenerate (longitudinally Lorentz invariant) Goto-Nambu type with fundamental tensor $\eta_{\sigma}^{\rho}$ and tension $T$ given by

$$
T=\kappa \lambda,
$$

since the vorticity $w$ is interpretable as due to a flux of $w / \kappa$ distinct microscopic vortices per unit area, where $\kappa$ denotes the constant momentum circulation associated with each individual vortex.

More particularly, for the specific model of the linearly separable kind characterised by (8.15) with the vorticity dependent factor $\Upsilon$ given by the linear formula (7.17) on which (8.22) is based, the tension of each such (degenerate Goto-Nambu type) string will be given by

$$
T=\kappa \mathcal{K} \Phi^{2}=\frac{\pi \hbar^{2}}{2} \Phi^{2} \ln \left\{\frac{w_{\odot}}{\langle w\rangle}\right\} .
$$

and in this case the perfect fluid contribution (A2) will be expressible simply by

$$
T_{\circ \sigma}^{\rho}=n^{\rho} \mu_{\sigma}+\left(\frac{1}{2} n \mu-V\right) g_{\sigma}^{\rho}
$$

which has just the form that would be obtained from (8.22) if the vorticity coupling coefficient $\mathcal{K}$ in it were set to zero.

Since the closure condition (3.7) gives the kinematic identity

$$
\nabla_{\nu}\left(w \mathcal{E}^{\nu \rho}\right)=0 \quad \Rightarrow \quad w_{\rho \sigma} \nabla_{\nu} w^{\nu \sigma}=w \perp_{\rho}^{\nu} \nabla_{\nu} w-w^{2} K_{\rho},
$$

where $K_{\rho}$ is the geometric curvature vector of the vortex sheet, as given ${ }^{[21]}$ in terms of its fundamental tensor (7.6) by

$$
K_{\rho}=\eta_{\sigma}^{\nu} \nabla_{\nu} \eta_{\rho}^{\sigma}=\mathcal{E}_{\sigma}^{\nu} \nabla_{\nu} \mathcal{E}_{\rho}^{\sigma},
$$

the proportionality relation (8.5) can be used to reduce the dynamical equation of motion (3.10) to the form

$$
\zeta_{\rho}=w\left(\perp_{\rho}^{\nu} \nabla_{\nu} \lambda-\lambda K_{\rho}\right)
$$

for any model of the subcategory characterised by (8.1). Since the vorticity $w$ is interpretable as due to a flux of $w / \kappa$ distinct string-like vortices per unit area, the Joukowski force density $\zeta_{\rho}$, as defined by (5.2), can be interpreted as representing a corresponding average Joukowski force per unit length, $Z_{\rho}$ say, acting on each individual vortex cell, that will be given by

$$
Z_{\rho}=\frac{\kappa}{w} \zeta_{\rho}=\frac{1}{2} \kappa \mathcal{E}^{\mu \nu} N_{\mu \nu \rho} .
$$

The equation of motion (A8) can thus be seen to be expressible as the condition that this Joukowski force per unit length should be given in terms of the corresponding effective vortex tension (A3) simply by

$$
Z_{\rho}+T K_{\rho}=\perp_{\rho}^{\nu} \nabla_{\rho} T .
$$

This can be recognised as a generalisation of the standard equation of motion for a global cosmic string in an axion field background ${ }^{[13][14]}$, to which it reduces when the tension $T$ is constant so 
that the gradient term on the right drops out. The constant tension case arises for the model (8.22) obtained from (7.17) to which (A4) applies when the equation of state is of the "stiff" type for which $\Phi$ is constant.

\section{Appendix B: The Lebedev Khalatnikov subcategory.}

It is easy to confirm that the category of theoretical models obtained by the convective variational approach described in Section 3 includes as a subcategory the class of models originally set up by Lebedev and Khalatnikov ${ }^{[8]}$. To show this it suffices to identify their generalised pressure function $\Psi$ with the Legendre transformed master function $\Psi$ on which the reformulated variational principle of the Section 4 is based, since on this basis our equation of motion (4.10) agrees precisely with the one that they obtained using a Clebsch type variational procedure. Instead of using a Lagrangian of the form (4.6) in which the master function is supplemented by the appropriate Kalb Ramond type coupling term, the variational formulation proposed by Lebedev and Khalatnikov used $\Psi$ by itself as Lagrangian, the cost of this apparent simplification being the need to consider the relevant momentum covector $\mu_{\rho}$ not as an independent field variable in its own right but merely as a derived quantity, specified by an appropriate set of Clebsch type potentials and their gradients. The question of whether one prefers to work with a set of scalar Clebsch type potentials as in the approach pioneered by Lebedev and Khalatnikov, or with a single antisymmetric tensorial potential of Kalb Ramond type as in the approach used here is merely a matter of taste and convenience in view of the ultimate agreement of the ensuing field equations. The Clebsch formulation has the technical advantage of using fewer independent field components but has the drawback that a larger number of different alphabetical letters are needed to characterise them. From the point of view of mathematical elegance and ease of formal manipulation, the Kalb Ramond formulation (either in its original version as given in the section 3 or the dual version of section 4) is more satisfactory: it involves a greater degree of gauge dependence but has the advantage of being more economical in its use of algebraic symbols.

Although the postulates of the Lebedev Khalatnikov theory are thus confirmed to be consistent with those adopted here, their category of models was restricted to a subclass of those considered in Section 4 by the supposition that the master function $\Psi$ depended only on $\mu$ and $h$ but not on the third independent scalar $w$, i.e. they assumed that the form of the master function would be characterised by

$$
\frac{\partial \Psi}{\partial w^{2}}=0
$$

Subject to this restriction the variation of $\Psi$ would be given simply by

$$
\delta \Psi=\ell_{\rho} \delta h^{\rho}-m^{\rho} \delta \mu_{\rho}
$$

with

$$
\ell_{\rho}=2 \frac{\partial \Psi}{\partial h^{2}} h_{\rho}, \quad m^{\rho}=2 \frac{\partial \Psi}{\partial \mu^{2}} \mu^{\rho} .
$$

In terms of these quantities, the stress momentum energy density tensor (5.11) will be reducible to the simple form

$$
T_{\sigma}^{\rho}=m^{\rho} \mu_{\sigma}+h^{\rho} \ell_{\sigma}+\left(\Psi-\ell_{\nu} h^{\nu}\right) g_{\sigma}^{\rho}
$$


which agrees with the expression given by Lebedev and Khalatnikov ${ }^{[8]}$. However a small discrepancy does occur in their expression for the modified current vector (4.11), which omitted the final term of the formula

$$
j^{\rho}=m^{\rho}-H^{\rho \sigma \nu} \nabla_{\sigma} \ell_{\nu}+2 w \ell_{\nu} \mathcal{E}^{\nu \rho}
$$

that we obtain in this case.

The choice of the particular kind of model introduced in Section 7 and Section 8 to match the results of our recent microscopic analysis ${ }^{[15]}$ for the case $\zeta=0$ was determined by the obvious ansatz that the master function $\Lambda$ should not depend on the Joukowsky scalar $\zeta$, but only on $n$ and $w$. We saw that in the weak vorticity limit under consideration, this ansatz was equivalent to the postulate that the conjugate master function $\Psi$ should depend only on $\mu$ and $w$ but not on the helicity scalar $h$. However there is not any obstacle to matching the same results ${ }^{[15]}$ by a model characterised by the alternative Lebedev - Khalatnikov ansatz to the effect that $\Psi$ should instead depend on $\mu$ and $h$ but not on $w$. This can be done simply by replacing $w$ in the deviation term given by (7.1) by the ratio $h / \mu$ which is the same as $w$ when $\zeta$ vanishes. It can be checked directly that the model specified by the deviation term

$$
\Psi \smile-\mathcal{K} \Phi_{\ominus}^{2} \frac{h}{\mu}
$$

that is obtained in this way will have a current vector and a stress momentum energy density tensor that agree with those obtained from the ansatz adopted in Section 7 for small $w$ provided $\zeta$ vanishes. However this ansatz leads to a more complicated form for the conjugate formulation which can be seen to have an explicit $\zeta$ dependence given by

$$
\Lambda^{\prime} \sim-\mathcal{K} \Phi_{\ominus}^{2} \sqrt{w^{2}-\frac{\zeta^{2}}{n^{2}}} .
$$

Until further evidence is provided by a more complete microscopic analysis, the criterion of simplicity would seem to rule against this latter model in favour of the separable model proposed in Section 7. However it may well turn out that neither alternative is adequate for cases in which $\zeta$ is too large to be neglected.

\section{References}

[1] G. Mendel, L. Lindblom, Ann. Phys. 205, 110 (1991).

[2] J.A. Sauls, in Timing Neutron Stars, ed H. Ogelman, E.P.J. Van Heuvel, pp 457 - 489 (Kluwer, Dordrecht, 1988).

[3] F. Lunde, T. Regge, Phys. Rev. D14, 1524 (1976).

[4] R.L. Davis, E.P.S. Shellard, Phys. Lett. B214, 219 (1988). 
[5] R.L. Davis, E.P.S. Shellard, Phys. Rev. Lett. 63, 2029 (1989).

[6] U. Ben-Ya'acov, Phys. Rev. D44, 2452 (1991).

[7] U. Ben-Ya'acov, Nucl. Phys. B382, 592 (1992).

[8] V.V. Lebedev, I.M. Khalatnikov, Sov. Phys. J.E.T.P. 56, 923 (1982).

[9] B. Carter, Class. Quantum. Grav. 11, 2013 (1994).

[10] Ya. B. Zel'dovich, Sov. Phys. J.E.T.P. 41, 1143 (1962).

[11] B. Carter, I.M. Khalatnikov, Phys. Rev. D45 , 4536 (1992).

[12] E. Witten, Phys. Lett. B153, 243 (1985).

[13] A. Vilenkin, T. Vachaspati, Phys. Rev. D35, 1138 (1987).

[14] M. Sakellariadou, Phys. Rev. D44, 3767 (1991).

[15] B. Carter, D. Langlois Phys. Rev. D, sous presse (1995).

[16] B. Carter, I.M. Khalatnikov, Ann. Phys 219, 243 (1992).

[17] J. Stachel, Phys. Rev. D21, 2171 (1980).

[18] P.S. Letelier, Phys. Rev. D20, 1294 (1979).

[19] W. Kopczynski, Phys. Rev. D36, 3582 (1987).

[20] B. Carter, Phys. Lett. B228, 446 (1989).

[21] B. Carter, Class. Quantum. Grav. 9, S19 (1992).

[22] A.F. Andreev, M.Yu. Kagan, Sov. Phys. J.E.T.P. 59, 318 (1982). 